\title{
Positionnement des systèmes multi-agents pour les systèmes de transport intelligents
}

\author{
F. Balbo ${ }^{1}$, E. Adam ${ }^{2}$, R. Mandiau ${ }^{2}$ \\ 1. Univ. Lyon, Mines Saint Etienne, \\ CNRS, Laboratoire Hubert Curien UMR 5516 \\ flavien.balbo@emse.fr \\ 2. LAMIH CNRS UMR 8201 \\ Université de Valenciennes \\ 59300 Valenciennes cedex 9, France \\ \{emmanuel.adam,rene.mandiau\}@univ-valenciennes.fr
}

\begin{abstract}
RÉSUMÉ. L'utilisation des nouvelles technologies de l'information et des communications pour l'amélioration des systèmes de transport est une solution devenue centrale dans le domaine du transport. Le résultat est ce que l'on appelle les Systèmes de Transport Intelligents (STI). Les enjeux sociétaux de leur mise en cuvre sont cruciaux en termes de réponse aux besoins des usagers mais également pour la conception de systèmes de transports durables. Dans ce papier, nous défendons l'idée que le paradigme multi-agent peut répondre aux besoins de ce domaine. Nous proposons ainsi de présenter le domaine des STI et de focaliser notre attention sur trois problématiques : la manière d'assurer sa fonctionnalité, la manière de le rendre plus «intelligent » malgré des contraintes de passage à l'échelle, et enfin ses conséquences éthiques. Quelques travaux de recherche illustrent notre propos.

ABSTRACT. The use of new information and communication technologies has become a central solution to improve transport systems. What has led to which is called the Intelligent Transport Systems (ITS). Societal challenges for ensuring their effective implementations are crucial both to respond to users needs, and to design sustainable transport systems. In this paper, we argue that multi-agent paradigm can address the needs of this domain. We introduce ITS, and we emphasize three main issues of ITS which are how to ensure its functionality, how to render it more "intelligent" despite constraints in scalability, and the ethical implications. Some selected research works are provided to illustrate these issues.
\end{abstract}

MOTS-CLÉS : Système de Transport Intelligent (STI), Système Multi-Agent (SMA), Trafic routier, Système d'Information des Voyageurs (SIV).

KEYWORDS: Intelligent Transport System (ITS), Multi-Agent System (MAS), Road traffic, Traveller Information Systems. 


\section{Introduction}

Des systèmes de transport performants ont un impact fort sur le développement d'une société. Rendre efficace le déplacement des biens et des personnes participe à la croissance économique et modifie nos territoires par l'amélioration de l'accessibilité (DGITM, 2011). Pour être durable, il faut que ces améliorations soient réalisées avec l'objectif de limiter les nuisances qu'engendrent l'augmentation de l'usage des transports. Ainsi, mieux se déplacer signifie également limiter l'impact environnemental de la pollution engendrée et limiter l'impact sociétal en améliorant la sécurité et les conditions de vie. La commission européenne a estimé que le transport contribue approximativement à $20 \%$ des émissions totales de $\mathrm{CO}_{2}$ en Europe (Todts, Calvo Ambel, 2015). Le transport est considéré comme l'un des principaux secteurs produisant des émissions polluantes et le seul secteur à croître de manière encore trop importante (EU, 2014a). Ces enjeux expliquent la raison pour laquelle de nombreux programmes de recherche à travers le monde visent à l'amélioration de nos systèmes de transport (en Europe, par exemple l'appel «Les transports intelligents, verts et intégrés » du programme H2020 (EU, 2014b)). C'est en effet une tâche difficile car la nature distribuée, ouverte, dynamique et partiellement contrôlable des réseaux de transport en font un domaine complexe.

L'utilisation des Nouvelles Technologies de l'Information et des Communications (NTIC) pour l'amélioration des systèmes de transport est une solution devenue centrale dans le domaine. L'augmentation de la puissance de calcul des ordinateurs ou des systèmes embarqués, ainsi que la qualité des capteurs de plus en plus sophistiqués, ont permis de proposer des mécanismes de contrôle plus efficace; ainsi qu'une meilleure prise en compte de l'opérateur ou des utilisateurs (Vanderhaegen et al., 2006 ; Boy, 2014). Le résultat est ce qu'on appelle des Systèmes de Transport Intelligents (STI). En France, les premiers STI sont apparus il y a une trentaine d'années avec la gestion centralisée des carrefours ou l'information sur le trafic Bison Futé. Si les STI améliorent la gestion des réseaux existants, ils ont également permis le développement de nouvelles solutions de transport comme le co-voiturage ou l'auto-partage.

Le terme générique STI recouvre une extrême hétérogénéité concernant les niveaux d'intelligence des systèmes. Par exemple, résoudre le problème de l'interopérabilité entre réseaux de transport pour assurer une solution billettique commune autorise un usage intelligent des réseaux en simplifiant les démarches des voyageurs mais le STI résultat n'est pas en soi un système intelligent. Une majorité de STI répondent à la définition de Jorion (1989) d'un système intelligent que l'auteur considère comme un «système interlocuteur susceptible de jouer vis-à-vis de son utilisateur le rôle de collaborateur intelligent». Ainsi, un système d'information sur le trafic résultant de la collecte de données issues de capteurs peut être considéré comme intelligent car il peut, à la manière d'un collaborateur, donner aux voyageurs ou gestionnaires de réseaux les informations pertinentes leur permettant de prendre des décisions intelligentes. L'intelligence de ce collaborateur peut croître en complexité en allant du traitement des données (e.g., Bison Futé), jusqu'à la conduite de véhicules autonomes en passant par la proposition d'alternatives (e.g., systèmes de navigation). A cette vision 
fonctionnelle de l'intelligence qui conduit à plus de délégation vers le «collaborateur », doit s'ajouter celle du support du service rendu. Ainsi dans un environnement complexe, il faut que le support du service soit capable de s'adapter et de passer à l'échelle. Il doit donc dans son fonctionnement même faire preuve d'une intelligence lui permettant de répondre à ces difficultés. Enfin, si l'objectif de développement d'un STI véritable «collaborateur intelligent » exhibant des propriétés d'autonomie, d'adaptation ou traitant des données personnelles est atteint alors la question de la gestion de cette intelligence se posera. Ainsi, la prise en compte de la dimension éthique des décisions ou plus généralement du comportement des STI devra être intégrée à leur fonctionnement.

Dans Bazzan et Klügl (2014), les auteurs présentent une synthèse des systèmes multi-agents (SMA) pour des applications essentiellement en transport et trafic routier. Pour notre part, nous proposons de considérer les approches multi-agents pour les STI, en prenant en compte d'autres éléments que l'aspect technologique du transport (e.g., la personnalisation de l'information pour les usagers, l'évaluation du risque d'événements perturbant le système dans sa globalité).

Dans cet article nous montrons comment le paradigme multi-agent peut être une réponse au développement d'un STI répondant à ces trois points de vue sur l'intelligence. Ainsi les propriétés d'autonomie, de réactivité, de communication, d'aptitude sociale et de pro-activité usuellement associées aux agents ont souvent été utilisées afin de développer des systèmes capables d'intelligence dans des environnements complexes. La rationalité des agents, la capacité de s'organiser afin de satisfaire un objectif peuvent servir de support au développement de collaborateurs intelligents capables de répondre aux besoins personnalisés et contextuels de leur utilisateur. Enfin, les interrogations quant au contrôle de cette délégation de notre autonomie à des entités intelligentes autonomes est déjà présente dans de nombreux travaux du domaine multi-agent. Après avoir discuté l'état d'avancement des STI selon ces trois points de vue, nous proposons d'illustrer par des travaux de recherche que nous avons entrepris, l'apport des SMA.

La suite de l'article est organisée de la manière suivante. En section 2, nous présentons le domaine des systèmes de transport intelligents, nous discutons les fonctionnalités attendues et objectifs à atteindre. En section 3, nous montrons comment l'intelligence déployée au sein d'un SMA permet la conception de STI dans un environnement complexe. Nous illustrons ce point de vue avec le problème de la gestion des informations voyageurs. Dans la section 4, nous discutons l'apport des SMA pour la conception d'un STI vu comme un collaborateur intelligent. Nous illustrons ce point de vue avec la gestion de trafic, et plus précisément comment les SMA peuvent aider à la gestion des intersections. Dans la section 5, nous montrons comment l'intelligence d'un STI amène de nouvelles questions notamment dans son interaction avec les humains. Nous illustrons cette problématique par les travaux sur le besoin d'éthique dans le déploiement de véhicules autonomes.

Afin de faciliter la lecture de l'article, nous proposons de définir, dans un glossaire situé en annexe, les principaux acronymes employés. 


\section{Présentation du domaine}

Dans le rapport européen (EU, 2012) concernant l'évaluation des programmes de recherche en transport, les Systèmes de Transport Intelligents (STI) sont considérés comme vitaux pour concevoir des systèmes de transport durables. Toujours selon ce rapport, par une intégration des technologies de l'information, des communications et du contrôle, les STI permettent aux autorités, opérateurs et individus de prendre de meilleures décisions. Les STI ne sont donc pas dédiés à un mode ou un service particulier mais concernent tous les systèmes qui améliorent l'utilisation des moyens de transport par l'utilisation d'un ensemble de technologies afin de satisfaire des objectifs du domaine. Dans la section 2.1 nous présentons rapidement les technologies clefs ainsi que les objectifs à atteindre puis une classification des systèmes obtenus. Nous montrons en section 2.2 en quoi il est difficile de concevoir et de mettre en œuvre un STI et en quoi le paradigme multi-agent est une réponse adéquate à ces difficultés.

\subsection{Définition d'un Système de Transport Intelligent}

Quelque soit la fonctionnalité associée au STI, il est construit à partir de données captées sur le réseau, qui sont reçues et traitées par un logiciel. Par conséquent, tous les progrès réalisés dans le domaine des communications, des capteurs et de l'informatique bénéficient potentiellement au domaine du transport. Par exemple, le développement des véhicules connectés ou autonomes n'est possible que par la mise en œuvre des communications entre véhicules et avec une infrastructure adaptée (e.g., grâce au standard IEEE 802.11p), le déploiement de capteurs performants (e.g., le Light Detection and Ranging - Lidar) (Li et al., 2014), et des capacités de calculs importantes pour par exemple, mettre en œuvre un module de l'architecture CALM (Ernst et al., 2009). L'utilisation combinée de ces technologies doit permettre de développer des STI pour des prises de décision mieux informées afin de (Figure 1) :

- Générer des gains économiques : Une meilleure connaissance du fonctionnement des réseaux permet de mieux utiliser les ressources, infrastructures et matérielles, déjà disponibles avec pour corollaire une diminution des temps de parcours. Cette amélioration donne directement des gains de productivité aux sociétés de transport mais également à tous les métiers dont une partie du temps de travail correspond à des déplacements comme les infirmières libérales par exemple. L'accroissement des informations disponibles permet enfin le développement de nouveaux modèles de mobilité et donc de nouveaux marchés notamment orientés vers l'économie du partage (Hussain et al., 2015). Enfin, l'amélioration de la logistique et notamment de la logistique urbaine (Faure et al., 2014) participe à la création de richesse. Par exemple le projet Logistique Urbaine Mutualisée et Durable (LUMD). (Hette et al., 2012) visait à concevoir une plateforme intelligente (adaptable aux événements et perturbations) de mutualisation d'offres de transport et de stockage de marchandises, particulièrement la livraison de l'ensemble de la presse papier dans la ville de Paris.

- Améliorer la sécurité : Le décideur bénéficie de cette connaissance pour mieux comprendre l'usage des réseaux sur du long terme et anticiper le traitement de nou- 
veaux évènements sur du court terme. Par exemple, l'information fournie par les systèmes de guidage des conducteurs de voiture leur permet d'adapter leur comportement à l'état du trafic ou à un plan de feux (Koukoumidis et al., 2012). De même, le transport de matière dangereuse peut être optimisé afin de limiter les risques comme dans le projet Geofencing-MD ${ }^{1}$.

- Améliorer la qualité de vie : La diminution des temps de parcours bénéficie aux voyageurs qui gagnent du temps personnel tout en diminuant le stress et la fatigue. L'évolution technologique rend possible l'accès à la mobilité pour de nouveaux publics. Par exemple, un meilleur accès à l'information pour les voyageurs facilite l'usage des transports publics et il rend possible leurs accès aux personnes déficientes (Banâtre et al., 2004).

- Améliorer la qualité de l'environnement : Son amélioration dépend essentiellement de la réduction des consommations d'énergie et de la pollution. Un moyen de réduire la pollution est d'envisager une utilisation accrue des véhicules électriques (Xiong et al., 2015), un autre moyen est de mieux utiliser les ressources. Si la réduction du temps de parcours participe à cette amélioration, c'est également le cas des solutions participant au report modal vers les transports publics. Par exemple, la gestion dynamique de la priorité aux carrefours à feux en faveur des bus améliore la qualité de service des transports publics sans trop détériorer la fluidité du trafic et renforce ainsi l'attractivité de ce mode (Bhouri et al., 2012).

Selon les modes et les fonctionnalités il existe de très nombreux STI. Dans (Giannopoulos, 2004 ; Nasim, Kassler, 2012 ; Rafiq et al., 2013), les auteurs proposent une définition par extension des fonctionnalités qu'un STI peut satisfaire. Une alternative pour définir un STI est de considérer le composant du système de transport qu'il concerne, i.e., pour lequel le STI fournit une fonctionnalité (Figure 1):

- Le bien ou le voyageur : Nous placerons dans cette catégorie les systèmes d'information voyageurs, de billettique et de logistique. Ces STI ont pour fonction de permettre une meilleure utilisation des réseaux existants par les voyageurs (transport de personnes) ou les biens (logistique). Par exemple, les STI pour la billettique facilitent le traitement des moyens de paiement et favorise l'intermodalité par une gestion tarifaire commune. La décision des autorités et exploitants d'une billettique commune doit être supportée par des solutions technologiques favorisant l'interopérabilité.

- La gestion des réseaux : Nous considérons dans cette catégorie les systèmes permettant la prise de décision sur le fonctionnement d'un réseau comme le Système d'Aide à l'Exploitation (SAE) pour le transport public (Balbo, Pinson, 2010) ou les systèmes de gestion de trafic (Papageorgiou et al., 2007). Selon l'échelle de temps considérée, la prise de décision concerne les autorités avec l'utilisation des informations collectées sur les réseaux pour le long terme jusqu'aux opérateurs de réseau pour la décision en temps réel dans un centre de régulation urbain par la mise en œuvre de stratégies de régulation du trafic.

1. http://www.transport-intelligent.net/champs-des-sti/gestion-des-flottes-et-du-fret/article/suivi-et -gestion-des-transports-de 


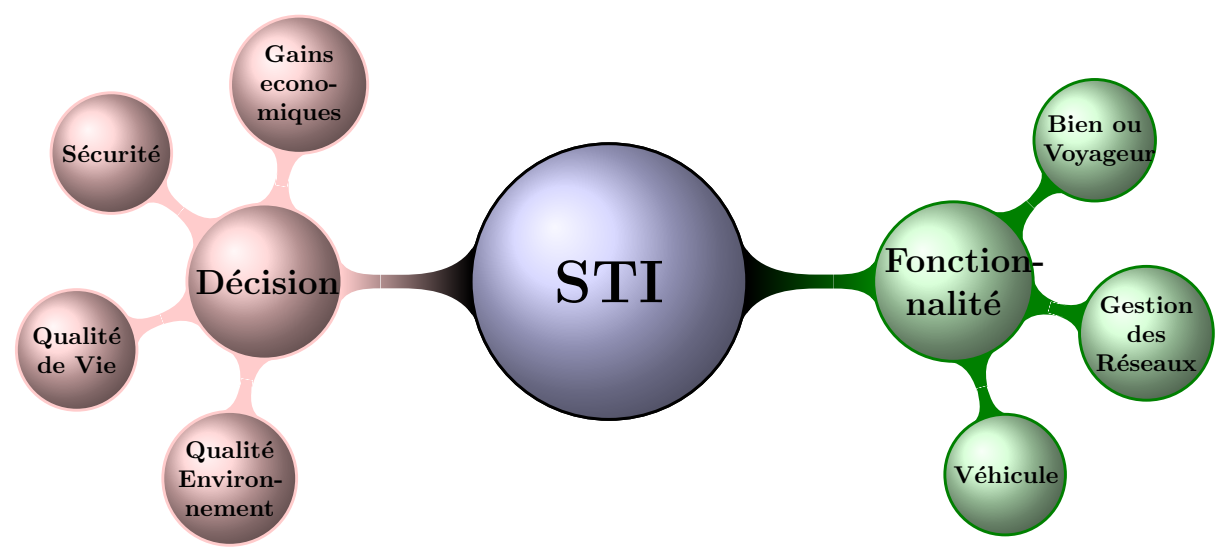

FIGURE 1. Principaux éléments pour les STI

- Le véhicule : Il s'agit d'améliorer la sécurité des déplacements par des aides à la conduite, une interaction accrue avec l'infrastructure et l'amélioration de la maintenance. L'aboutissement de cette évolution est le développement des véhicules autonomes (Dafflon, Gechter, 2014; Silberg et al., 2012; Anderson et al., 2014) pour lesquels de nombreux travaux sont actuellement en cours. Ces STI devraient permettre une réduction drastique du nombre d'accidents en éliminant les risques liés au facteur humain. Ils devraient accroître la mobilité en permettant à de nouveaux publics comme les handicapés, jeunes ou encore non titulaires d'un permis de se déplacer. De plus, il y aurait moins de congestion avec une diminution de l'espace inter-véhiculaire et leur coût serait réduit en permettant aux voyageurs d'utiliser ce temps pour d'autres occupations. Enfin, l'espace pourrait être utilisé autrement en permettant : 1) la densification des voies utilisées pour le transport et donc d'occuper moins d'espace au sol; 2) le développement de nouveaux modèles de mobilité comme l'utilisation des parkings extérieurs au ville et des navettes automatiques en centre ville et rendant possible par exemple la diminution des parkings des centres d'affaire qui occupent $31 \%$ de l'espace (Anderson et al., 2014).

Après avoir montré l'importance du développement de Systèmes de Transport Intelligents performants et la grande richesse des champs d'application, nous montrons dans la section suivante quels sont les principaux défis pour atteindre les objectifs fixés.

\subsection{Défis de conception d'un STI}

L'utilisation des NTIC dans le domaine du transport est une tâche particulièrement difficile. Il faut en effet développer un système pouvant assurer une fonctionnalité dans un environnement complexe, en interagissant avec de nombreux systèmes existants et 
capable d'intégrer à la fois des intérêts collectifs et individuels. A l'instar d'autres chercheurs (Bazzan, Klügl, 2014), nous pensons que l'utilisation des systèmes multiagents peut réellement apporter des solutions aux défis posés par le développement des STI.

La première difficulté concerne le fonctionnement d'un réseau de transport qui est complexe par nature car dynamique, ouvert, incertain et potentiellement à large échelle. Cette caractéristique est commune à tous les modes car tous les véhicules qui se déplacent sur un réseau, même s'ils le font dans un site protégé comme par exemple le transport souterrain, sont soumis à des aléas qui vont affecter son comportement comme des pannes ou incidents de personnes. Le STI doit donc intégrer dans son fonctionnement sa relation à un environnement complexe à partir duquel les informations sont issues et où les actions sont réalisées. Les propriétés de pro-activité, d'autonomie et de rationalité associées aux agents intelligents peuvent être utilisées afin de pallier les difficultés liées à la dimension complexe du domaine. Par exemple dans un SIV (Zargayouna et al., 2006), ces propriétés sont utilisées afin de trouver dynamiquement les informations pertinentes pour un voyageur lors de son déplacement y compris en cas de perturbation.

La seconde difficulté concerne la nécessité de résoudre les problèmes d'interopérabilité liés au fonctionnement du STI. Un STI doit être interopérable avec les STI ${ }^{2}$ qui participent à son fonctionnement, avec les STI qui utilisent ses fonctionnalités ou au contraire lui en fournissent. Il existe différents niveaux d'interopérabilité interdépendants pour un système de transport. Ainsi J.F Jannin en présente cinq ${ }^{3}$ qui sont respectivement les niveaux Politiques, Légaux, Organisationnels, Sémantiques et Techniques. Les NTIC concernent directement les deux derniers niveaux. Au niveau sémantique, l'utilisation d'ontologies est pertinente pour les STI (Oliveira et al., 2013), car elles permettent la définition et l'utilisation d'un vocabulaire et d'un langage commun aux différents agents impliqués dans le STI. Ainsi dans Moraitis et al. (2005), la connaissance des agents pour des services de transport est formalisée sous forme de graphes «Resource Description Framework (RDF)». Ainsi chaque fournisseur de service utilise sa propre ontologie pour décrire son service lors de ses communications et fournit un fichier au standard «Ontology Web Language (OWL)» qui pourra être utilisé afin de rechercher une correspondance entre ses concepts avec ceux de ses interlocuteurs (i.e., broker).

Au niveau technique, le paradigme multi-agent peut également apporter toute son expertise. Si l'utilisation de normes et de standards sont indispensables à une compréhension commune des données et des comportements attendus des différents systèmes, le paradigme multi-agent apporte en plus une vision unificatrice (Camus et al., 2015). Ainsi, dans l'exemple test sur l'interopérabilité entre plateformes multi-agents proposé par la «Foundation for Intelligent Physical Agents (FIPA)» (Poslad, 2007) pour

2. Par souci de simplification, nous utilisons ici le terme STI pour tout système d'information dédié au transport.

3. http://www.cotita.fr/IMG/pdf/01_DGITM_MTI_R_PAGNY_Enjeux_ITS.pdf 
un SIV (Núñez-Suárez et al., 2000), la notion unique d'agent recouvre des composants hétérogènes. L'utilisation des concepts de haut niveau du domaine des systèmes multi-agents donne une vision unifiée du système. En effet, les travaux sur les interactions ou l'organisation permettent d'introduire des concepts de plus haut niveau qui participent à la mise en œuvre de l'interopérabilité en formalisant les relations entre les composants hétérogènes (les agents logiciels, et également des services Web).

La dernière difficulté concerne la nécessité de prendre en compte simultanément des intérêts collectifs et individuels. Le domaine du transport est un des seuls domaines où des individus de tout horizon utilisent un bien partagé qu'est l'infrastructure de transport utilisé et doivent interagir sans même se connaître. Il faut donc pouvoir gérer les conflits préservant les intérêts individuels et collectifs. Cela suppose de parvenir à prendre en compte les attentes des voyageurs sans nuire au fonctionnement global du réseau et réciproquement. Par exemple dans le domaine du trafic, le code de la route et l'utilisation des feux de signalisation sont des solutions qui règlent les conflits selon un point de vue collectif sans prendre en compte les besoins individuels. Le résultat est un système connu comme inefficace au niveau individuel et collectif. A l'inverse, dans le projet européen Instant Mobility ${ }^{4}$, l'objectif est de prendre en compte les attentes des voyageurs afin d'assurer au mieux leur déplacement en assurant une optimisation de l'adéquation de l'offre à la demande. Le problème est que l'efficacité de cette solution est au détriment du respect de la vie privée des voyageurs puisque l'hypothèse de fonctionnement est une localisation en temps réel. Les travaux dans le domaine multi-agent ont permis d'approfondir et d'étudier les effets du comportement individuel d'un agent (l'entité de base) sur le comportement collectif, et vice-versa. Les SMA constituent donc une solution pour gérer les conflits entre intérêts collectifs et individuels dans un système de transport.

\section{Les SMA comme support au fonctionnement d'un STI}

Cette section présente comment le paradigme multi-agent a été utilisé pour un STI afin d'assurer leur fonctionnalité dans un environnement complexe. Nous proposons d'illustrer ces difficultés par des exemples issus du sous-domaine des SIV.

Un SIV doit aider le voyageur dans son choix modal et lui faciliter l'usage des réseaux. Par conséquent, un SIV doit proposer de l'information pré-voyage en donnant l'offre globale de tous les modes de transport sur un déplacement donné. Le SIV doit également donner de l'information pendant le voyage en accompagnant le voyageur et en l'informant à bon escient. La conception d'un SIV remplissant ces objectifs aura pour avantage d'accrôitre la confiance de l'usager dans son usage des réseaux et constitue un facteur de fidélisation. Dans la section 3.1, nous montrons comment les différentes étapes dans le fonctionnement d'un SIV bénéficient des travaux dans le domaine multi-agent. Dans la section 3.2, nous montrons comment un SMA peut être utilisé pour permettre l'usage d'une masse d'informations dynamiques.

4. http://www.instant-mobility.com/ 


\subsection{Défis des Systèmes d'Information Voyageur}

Un SIV permet aux voyageurs d'accéder aux informations nécessaires à l'usage des réseaux parcourus lors de leur déplacement. Cette classe de système est donc une interface entre les réseaux producteurs d'informations et les voyageurs qui les consomment. Ces informations doivent être collectées à partir d'un environnement complexe, puis être traitées par le SIV afin qu'il rende le service attendu et enfin être accessibles par les voyageurs selon leurs besoins. Chacune de ces étapes correspond à des enjeux spécifiques pour lesquels le paradigme multi-agent peut apporter des solutions.

La phase de collecte est souvent considérée comme du domaine des capteurs, l'enjeu étant alors d'améliorer leur performance et ainsi la qualité des informations obtenues. Le coût des capteurs baissant, ils sont déployés à grande échelle et équipent aussi bien l'infrastructure que les véhicules. Cependant, si les données sont dorénavant accessibles, il faut dans un environnement complexe être sûr de leur qualité pour obtenir de l'information. Pour Vinyals et al. (2011), les SMA sont une approche pertinente pour modéliser les réseaux de capteurs et réutiliser les solutions du domaine. Ainsi dans Chen et al. (2009), l'approche multi-agent est utilisée afin de valider le fonctionnement des capteurs. Pour l'estimation du trafic sur une autoroute, les auteurs proposent d'utiliser des agents mobiles qui, d'une station de recueil à l'autre, vont valider localement les résultats fournies par les capteurs. Dans Ando et al. (2006), les véhicules sont des capteurs mobiles et l'utilisation de phéromones sur une carte virtuelle permet de donner un état du trafic. Le modèle d'interaction permet l'agrégation des données fournies pour obtenir une information à disposition du conducteur. L'approche multi-agent peut donc être utilisée avec succès afin de pallier les difficultés pour obtenir une information de qualité dans un environnement complexe. Nous détaillons en section 3.2 une application d'information voyageurs pour des places de stationnement qui illustre l'apport des SMA pour répondre à cette difficulté.

L'information étant disponible, il faut construire le SIV qui la mettra à la disposition des voyageurs. La difficulté est de fournir la bonne information au bon moment au bon voyageur. Atteindre cet objectif suppose de prendre en compte des informations sur le voyageur et d'assurer un suivi tout au long du déplacement. Les systèmes de guidage des conducteurs répondent à ces spécifications. L'approche multi-agent doit faciliter la personnalisation du service tout en respectant la sphère privée du voyageur. Dans Radu et al. (2013), un agent assistant de conduite peut apprendre le profil du conducteur, prendre en compte son agenda et combiner ces informations avec l'état du trafic afin de lui proposer le meilleur itinéraire. Dans Repenning et Ioannidou (2006), un système multi-agent informe en temps réel un voyageur handicapé en l'avertissant par exemple de l'arrivée de son bus par une notification compatible avec son handicap.

Le système d'information voyageur construit, il est utilisable pour le réseau ou pour un ensemble de réseaux. Cependant, les déplacements étant multimodaux et les informations annexes à prendre en compte multiples comme la météo ou les points d'intérêt, le voyageur doit pouvoir être aidé afin de trouver l'information pertinente 
parmi l'ensemble des SIV et autres services. Par exemple, lorsque le SIV concerne différents réseaux comme Google Transit ${ }^{5}$ ou Rome 2 Rio $^{6}$, le service de calcul d'un itinéraire multimodal est fondé sur l'agrégation des données collectées auprès de ces réseaux. Il n'est pas personnalisé et ne prend pas en compte d'autres sources d'information ou services. L'approche multi-agent permet d'adopter un autre point de vue en considérant un SIV multimodal et multi-services comme le résultat de la réutilisation coordonnées des SIV existants. Dans les travaux issus de la FIPA (Núñez-Suárez et al., 2000 ; Coyle et al., 2002), l'organisation d'un déplacement est considérée comme un appel d'offre de services. Chaque agent représente un SIV d'un réseau de transport ou le système d'information d'autres services comme la réservation d'hôtel et ils négocient pour répondre à la requête. Le SMA recherche les services pouvant répondre à la requete de l'utilisateur et propose des mécanismes de coordination (Moraitis et al., 2005 ; Petit-Rozé et al., 2004).

\subsection{Illustration de l'utilisation de SMA comme support à un système d'information voyageur}

Nous illustrons l'apport des SMA en proposant deux exemples illustrant notre propos, à savoir sur l'usage dynamique pour la recherche de places de stationnement (3.2.1) et l'usage personnalisée des informations et des services (3.2.2).

\subsubsection{Faciliter l'usage d'une masse d'informations dynamiques}

Une partie importante des nuisances concernant le trafic urbain est due au «trafic de recherche », qui est celui généré lors des recherches de places de stationnement. D'après une étude (Shoup, 2006), $40 \%$ des véhicules en circulation cherchent un stationnement dans une zone urbaine. Ce trafic de recherche entraîne pour près de 10 millions d'heures perdues par an en France (Nangeroni, 2010). La conception d'un STI dédié à la gestion des places de stationnement doit permettre de limiter le coût de cette activité inutile. Bessghaier et al. (2012) présentent une solution multi-agent allant des collectes d'informations aux prises de décisions coordonnées entre conducteurs.

Il existe des problématiques propres à chaque type de stationnement et en particulier sur le stationnement de rue qui illustre le mieux la difficulté d'obtenir une information de qualité. L'information peut être collectée de différentes manières. Il peut s'agir de capteurs fixes, dans la chaussée ou des caméras, ou mobiles, des véhicules roulants sont équipés pour analyser l'espace disponible entre les véhicules stationnés. L'information peut également provenir des conducteurs eux mêmes. L'utilisation de capteurs supposent de trouver un compromis entre un coût matériel et une information disponible uniquement où le déploiement est réalisé. La plupart des solutions existantes centralise les informations sur la disponibilité des places de stationnement

5. http://maps.google.com/landing/transit/index.html

6. http://www.rome2rio.com/ 
et les demandes des conducteurs voir leur localisation en temps réel. Ce choix pose le problème du passage à l'échelle et le respect de la vie privée. Enfin, il faut accompagner la gestion de cette information d'un mécanisme d'attribution car la seule diffusion de l'information sur les places peut avoir un résultat contre-productif avec une concentration du trafic.

Pour répondre à ces contraintes, Bessghaier et al. (2012) proposent un système multi-agent composé d'agents assistant des conducteurs au sein d'une communauté. Le trafic est composé de conducteurs (membres de la communauté) et d'autres avec lesquels ils sont en compétition pour la recherche de places de stationnement. Chaque assistant a deux tâches distinctes, la première est de participer à la gestion des informations disponibles sur le stationnement, la seconde est de guider le conducteur vers la place la plus satisfaisante pour lui. L'agent assistant doit donc être coopératif pour le partage des informations et compétitif pour l'accès aux ressources, le néologisme de coopétitif (Y. Luo, 2007) est donc repris pour qualifier son comportement.

Les agents communiquent en utilisant un réseau inter-véhiculaire - en anglais Vehicular Ad Hoc Networks (VANETs) - (Bessghaier et al., 2012), afin de n'utiliser aucune infrastructure et d'assurer un traitement local de l'information. Le principe est de faire réaliser par les assistants une diffusion régulière de leurs informations. La diffusion est limitée dans l'espace et le temps grâce à une limite temporelle de validité de l'information. Ainsi l'information est physiquement disponible dans l'espace qui peut être parcouru par les véhicules par lesquels l'information transite avant qu'elle ne soit périmée. La limite temporelle doit être fixée afin de prendre en compte les caractéristiques de la place. Ainsi si la demande est très forte comme en centre ville, l'information sur la place sera vite périmée alors qu'elle peut être plus largement diffusée en périphérie. Chaque agent qui quitte ou occupe une place connue comme libre diffuse cette information. Les agents échangent de l'information sur la disponibilité des places mais également sur leur occupation. L'objectif est de gérer le cas ou une place est connue comme libre et qu'un membre de la communauté la trouve occupée. Chaque agent avec ces informations vérifie la validité de sa propre connaissance et diffuse le résultat de sa mise à jour.

Lorsqu'un conducteur est à proximité de sa destination, le comportement de l'assistant devient compétitif. Cela signifie qu'il ne va plus partager l'information concernant la place qui est choisie par le conducteur. L'objectif est de limiter sa diffusion et donc le nombre de concurrents. Si le conducteur est guidé par l'assistant vers la ressource, il peut également choisir une autre place rencontrée lors de son déplacement. Les expérimentations ont montré l'efficacité de la proposition par rapport aux véhicules non membres de la communauté avec une diminution d'en moyenne $50 \%$ du temps de recherche. Bessghaier et al. (2012) montrent également que l'intérêt d'un SIV pour le stationnement atteint un maximum mesuré en termes de taux de succès (i.e., le ratio de places trouvées grâce au système) selon la rareté des places. En effet, s'il y a une forte demande alors il y a trop de décalage entre la valeur de l'information et l'état de la place. 


\subsubsection{Faciliter l'usage personnalisée d'une masse d'informations et de services hétérogènes}

Un SIV doit proposer de l'information pré-voyage en donnant l'offre globale de tous les modes de transport sur un déplacement donné. Le SIV doit également fournir de l'information pendant le voyage en accompagnant le voyageur et en l'informant à bon escient. Il est ainsi possible de définir un ensemble de fonctions qu'un agent peut accomplir pour aider un utilisateur (Petit-Rozé et al., 2004). Sa première fonction est de collecter l'information sur des systèmes hétérogènes et distribués. En effet, l'utilisateur doit pouvoir formuler son besoin sans connaître les sources d'information pouvant y répondre. Afin de constituer une réponse personnalisée, la seconde fonction consiste à intégrer les données obtenues. Le voyageur doit pouvoir spécifier ses préférences et obtenir une (des) réponse(s) en conséquence. Enfin, la dernière fonction consiste à assurer le suivi des informations afin de surveiller le bon déroulement du déplacement. Le voyageur doit pouvoir être averti de tout évènement susceptible d'interférer avec son déplacement.

Pour cette dernière fonctionnalité, Zargayouna et al. (2006) conçoivent un Serveur Agent d'Information Voyageur qui à partir de l'itinéraire d'un voyageur identifie les évènements qui peuvent perturber son déplacement (Figure 2). La proposition s'appuie sur le modèle EASI (Saunier et al., 2014) qui permet à des agents cognitifs d'utiliser l'environnement pour échanger des messages. Le modèle EASI permet une mise en commun des informations disponibles et mise à jour par les serveurs. Il ne s'agit pas de dupliquer les données proposées par des serveurs d'information mais d'organiser leur utilisation dans un contexte défini. Ce dernier étant défini par des filtres déposés/retirés dynamiquement de l'environnement par les agents. Dans cette étude, les services recherchés par les utilisateurs (e.g., la recherche d'un itinéraire ou des informations sur le trafic) sont accessibles par ce mécanisme de filtrage.

\section{Les SMA pour un passage à l'échelle des STI}

Nous présentons dans cette section comment le paradigme multi-agent peut être utilisé afin de mettre de l'intelligence dans le service rendu par un Système de Transport Intelligent, en prenant en compte la dimension du passage à l'échelle. Nous proposons d'illustrer les difficultés par des exemples issus du trafic routier.

Le passage à l'échelle est un élément nécessaire pour la conception d'un STI, à la fois par le nombre d'usagers croissants et par le nombre d'informations pouvant être exploitées. La congestion de trafic résultant de ce problème de passage à l'échelle est discutée en section 4.1. Dans la section 4.2, nous détaillons pour deux problématiques différentes des solutions multi-agents mises en œuvre pour supporter un fonctionnement intelligent. 


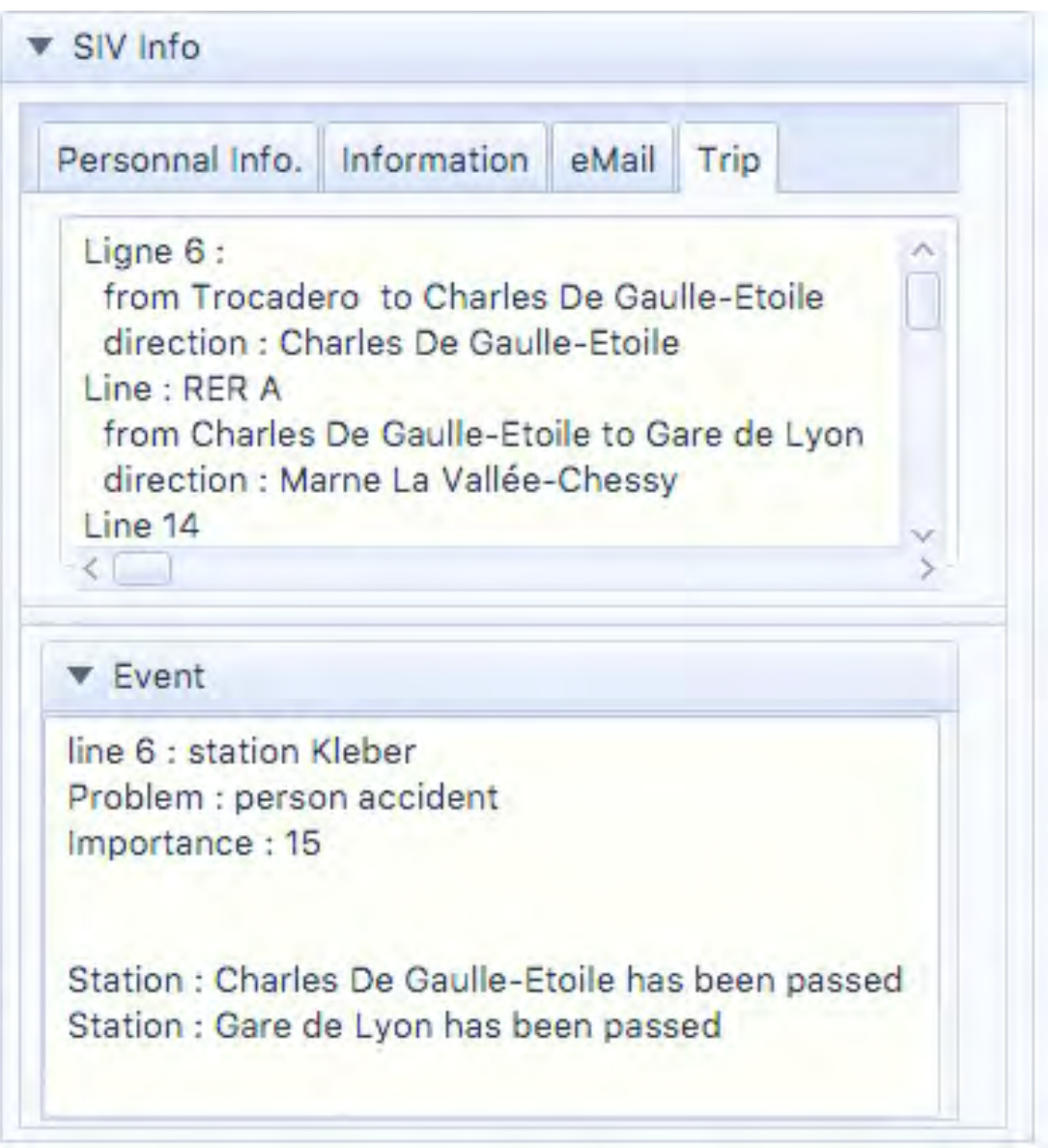

FIGURE 2. Une Interface SIV alertant d'un événement imprévu dans le déplacement d'un usager

\subsection{Défis du passage à l'échelle}

La congestion de trafic dans les villes est un problème difficile à résoudre. Elle est provoquée lorsque le flux de trafic dépasse les capacités typiques de l'infrastructure routière. Plus clairement, elle est causée par des événements imprévus (accidents, travaux) ou tout simplement par un nombre d'usagers croissants. Elle peut générer des « remontées de queues », produisant à leur tour des situations bloquantes.

La détection de ces zones critiques et une bonne évaluation a priori du nombre d'usagers peuvent contribuer à réduire ces congestions. Malheureusement, la régulation classique du trafic dans ce cas, n'est pas suffisante. Il apparaît alors nécessaire 
de prendre en compte des informations trafic temps réel. Par exemple, des approches telles que Spit Cycle Offset Optimisation Technique (SCOOT) (Hunt et al., 1982) ou Sydney Coordinated Adaptive Traffic System (SCATS) (Lowrie, 1990) utilisent des informations temps réel pour adapter les feux de signalisation aux carrefours. Les informations de trafic ont été collectées par des capteurs magnétiques et caméras vidéos, pour obtenir le nombre de véhicules approchant ou sortant d'un carrefour.

De nouvelles approches ont pris en compte des technologies de communication sans fil, e.g., Radio Frequency Identification (RFID) (Wen, 2010) et Internet of Vehicles (IoV) (Wunderlich et al., 2008), et fournissent des informations trafic plus fines en supportant une interaction directe. Toutefois, la masse d'informations manipulées sous contraintes temporelles présuppose des modèles multi-agents pouvant aller du filtrage des informations reçues (Ksontini et al., 2015) à des modèles de régulation de trafic résultant d'une coordination locale (Bhouri et al., 2012 ; Guériau et al., 2015; Goodall et al., 2013 ; Pandit et al., 2013).

Comme nous l'avons par ailleurs évoqué, le nombre d'informations à exploiter (dynamiques et hétérogènes) nous permet d'envisager des améliorations des conditions de déplacements des usagers. Cet apport est réellement possible à la fois par une prise en compte des évolutions matérielles (4.2.1) et la capacité de proposer des décisions « intelligentes » par des approches de coordination (4.2.2).

\subsection{Illustration de la gestion intelligente de trafic}

\subsubsection{Faciliter l'échange d'informations par la prise en compte des aspects matériels}

Pour les différents modes de transport, les évolutions technologiques ont accéléré les déplacements des usagers sur de plus longues distances tout en améliorant la sécurité et le confort des usagers. Par exemple, les conducteurs automobiles peuvent se servir de différents systèmes d'assistances tels que les systèmes de freinage, de régulation de vitesse, voire les systèmes de parking automatique. Utilisant des réseaux de communication standards (e.g., IEEE 802.11 ou UWB) (J. Luo, Hubaux, 2005), les véhicules peuvent émettre ou recevoir des informations de leurs voisins. Il est alors possible de prévenir les autres véhicules d'un danger immédiat ou d'un ralentissement, et transmettre des informations plus difficiles à évaluer comme des conditions de trafic en temps réel. Dans des situations de danger, le conducteur prévient les véhicules qui sont derrière lui, par les warnings. Dans des situations où l'information nécessite une évaluation macroscopique (densité du trafic) - i.e., une mesure qui ne situe pas simplement au niveau du conducteur (point de vue microscopique), mais au niveau d'un groupe de conducteurs - des informations échangées (via des VANETs) peuvent être nécessaires sur des portions de routes plus importantes et augmentent ainsi la sécurité des conducteurs (Kakkasageri et al., 2013).

Pour répondre notamment à cette exigence de pouvoir échanger des informations sur des distances de route plus importantes, alors que la portée de ces réseaux reste relativement peu élevée, le projet VESPA (Delot et al., 2009 ; 2010) vise à propo- 
ser une architecture embarquée dans les véhicules, permettant de partager des informations entre ces derniers. L'objectif consiste alors à faciliter la communication des informations liés à des événements temporaires et localisés (e.g., freinage d'urgence, recherche de place de parking), tout en prenant en compte l'utilité des données pour chaque conducteur. Le système évalue selon un modèle probabiliste la pertinence de l'information pour un conducteur donné (une estimation temporelle de rencontre entre la position de l'événement détecté, ou de sa trajectoire si celui-ci est mobile, et celle du conducteur). Il évalue l'importance de l'événement à transmettre, et détermine la zone impliquée par l'événement (zone où l'information est utile aux véhicules).

\subsubsection{Faciliter la coordination dans la prise en compte des aspects décisionnels}

Dans l'élaboration du processus décisionnel, il est également important de s'intéresser au difficile problème de la coordination (Bonhomme et al., 2015 ; Gechter et al., 2012 ; Guériau et al., 2015; Tlig et al., 2014). Plusieurs définitions en ont été données dans la littérature (Lesser, 1998; Malone, 1998), reprises également dans (Guessoum et al., 2012), chacune mettant en évidence un point de vue spécifique. La coordination est le processus qui permet à un agent de raisonner sur ses propres actions et les actions (anticipées) des autres agents, pour essayer de garantir que le groupe d'agents agit de manière cohérente. Jennings (1996) souligne que le processus de raisonnement ne devrait pas prendre en compte que les états courants du système, mais aussi les états futurs.

Le trafic routier peut être défini comme un ensemble de phénomènes résultant des déplacements des usagers de la route sur un réseau routier à capacité limitée. Le «système de trafic » est caractérisé par une offre constituée d'une infrastructure routière conçue pour répondre à un optimum collectif et par une demande toujours croissante des usagers qui cherchent à atteindre un optimum individuel. La conciliation de l'intérêt collectif et de l'intérêt individuel a engendré de nombreuses recherches, notamment sur la supervision de trafic, l'optimisation de flux de trafic et l'étude de nouvelles infrastructures routières (Bazzan, 2005 ; Hall, 1997 ; Lieberman, Rathi, 1997 ; Reece, Shafer, 1993 ; Ruskin, Wang, 2002) ainsi que le développement d'outils commerciaux comme Vissim (Vissim, 2005).

Le postulat sur lequel repose l'approche multi-agent est le suivant : le trafic est le résultat de la somme de toutes les actions et interactions des différents acteurs du trafic : usagers (automobilistes, piétons, deux roues), signalisation, infrastructures routières qui essaient de se coordonner en essayant de modéliser assez finement le comportement des agents (Trannois et al., 1998; Paruchuri et al., 2002; Mandiau et al., 2012 ; Espié, 1995). L'approche comportementale de la simulation multi-agent s'attache donc à modéliser et à reproduire le plus fidèlement possible les comportements et interactions des différentes entités simulées de manière à obtenir des phénomènes de trafic réalistes. Ceux-ci sont dits émergents et dépendent d'une distribution de comportements (hétérogénéité des pratiques individuelles) parmi un ensemble de véhicules simulés sur un réseau routier. 


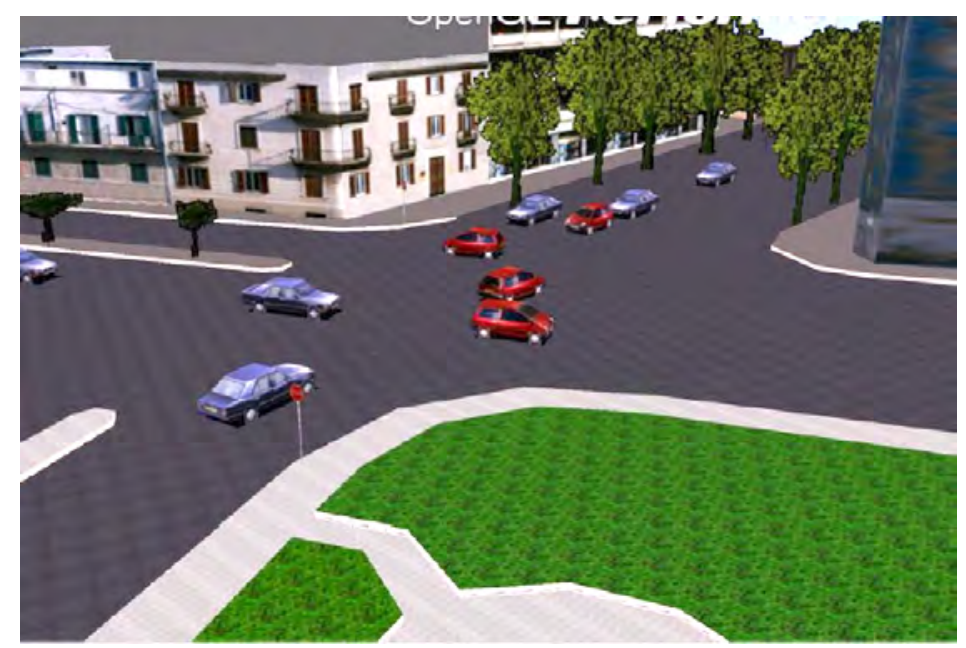

FIGURE 3. Carrefour Roma-Zerbi : un exemple d'intersection simulé par une approche multi-agent (simulateur ArchiSim)

Un des problèmes réputés théoriquement difficile dans le développement d'outils de simulations est le cas de la gestion d'intersections. Par exemple, le carrefour complexe Roma-Zerbi, présenté en Figure 3, a permis notamment de valider l'approche multi-agent dans un contexte réel sur un simulateur de trafic routier, appelé ArchiSim (Espié, 1995). Dans ce contexte, le débit réel est comparé au débit obtenu par simulation sur les différents axes (Mandiau et al., 2008). L'idée initiale de Champion (2003) consiste à considérer une situation de trafic à une intersection comme un jeu (Prentice, 1974). Un jeu (Guerrien, 1997) est une situation pour laquelle des individus (les joueurs) doivent choisir parmi un certain nombre d'actions (stratégies) possibles dans un format prédéfini (les règles du jeu). Le résultat de ces choix donne une sortie du jeu (la solution), à laquelle est associée un gain positif ou négatif pour chacun des participants. Il a été pris comme hypothèse que chaque joueur peut potentiellement jouer un jeu différent des autres (Champion, 2003), notamment car chaque agent perçoit seulement une partie de la situation globale. Par exemple, un conducteur peut considérer qu'il a la priorité vis-à-vis d'un autre conducteur; mais le second conducteur ne perçoit pas nécessairement sa relation avec le premier de la même manière. La recherche des stratégies gagnantes (Mandiau et al., 2008) a été modélisée par la perception des priorités reliant les agents deux-à-deux. La matrice de jeu caractérisant une situation complexe de carrefour ainsi définie par les différents agents a permis de proposer des mécanismes de raisonnements plus réalistes et passant à l'échelle. 


\section{Les SMA pour la prise en compte des risques et de l'éthique dans un STI}

Nous avons dans les sections précédentes pu apprécier l'usage des SMA pour améliorer le fonctionnement des STI en prenant en compte le problème du passage à l'échelle. Au delà de ce paradigme, l'amélioration des capacités de ces systèmes a pour corollaire une amélioration de leur autonomie et une intégration plus élevée. Il devient alors nécessaire de s'interroger sur les conséquences d'une telle évolution. Nous discutons dans la section 5.1, des risques qu'entrainent le développement d'un STI et illustrons dans la section 5.2, des exemples de mise en œuvre de solutions multi-agents.

\subsection{Risques d'une évolution incontrôlée}

Comme le montre Moor (2006), il devient nécessaire de ne plus seulement évaluer les systèmes produits selon leur capacité à atteindre les objectifs qui lui sont fixés mais également selon la manière dont ils sont atteints. La norme d'évaluation n'est alors plus seulement technologique mais également éthique. De plus en plus de chercheurs en intelligence artificielle se sentent concernés par ces enjeux (Russell et al., 2015). Par exemple, le projet ANR «eThique et Agents autonomes (eThicAa) ${ }^{7}$ a pour objectif de définir ce que devrait être un système composé d'un ou plusieurs agents, impliquant des humains, et capable de gérer des conflits éthiques, aussi bien au niveau individuel qu'au niveau collectif. Cette problématique concerne tout particulièrement le transport puisque le fonctionnement des systèmes dédiés impacte directement l'humain.

Nous retrouvons cette problématique quel que soit le destinataire du système. Par exemple, un SIV intelligent, au sens de Jorion (1989), sera le collaborateur d'un voyageur. Il peut être amené à prendre des décisions à sa place et donc assumer une part de responsabilité ou utiliser des informations qui relèvent de sa sphère privée. On retrouve au niveau de l'opérateur d'un réseau de transport le même questionnement concernant la collecte des informations ainsi que la prise de décisions qui pose également des problèmes de gestion de la vie privée et de la responsabilité (Steinfeld, 2010). Par exemple, Casey (2003) utilise les informations GPS issues des téléphones des voyageurs d'un bus afin de les localiser en temps réel. Si l'efficacité de la solution doit être discutée, il en est de même des problèmes éthiques de suivi et de respect de la vie privée qu'elle pose.

Enfin le développement des véhicules autonomes illustre également cette évolution technologique et concentre les interrogations. Ainsi selon l'association des ingénieurs automobiles américaines (Dokic et al., 2015) ou la National Highway Traffic Safety Administration (NHTSA), nous sommes entre le niveau 2 (le véhicule est équipé d'une combinaison de services permettant le contrôle de la vitesse et de la voie) et 3 (le véhicule perçoit son environnement et alerte l'humain en cas de problèmes pour qu'il

7. http://ethicaa.org/ 
intervienne en quelques secondes) dans leurs échelles respectives de l'autonomie des véhicules. Au niveau le plus élevé, le conducteur pourrait s'endormir, le véhicule prenant lui même ses décisions. Le dernier niveau suppose que le véhicule connaisse la destination des passagers et il faut assurer que cette information demeurera dans leur sphère privée (Glancy, 2012). De plus, la conduite du véhicule autonome dans le trafic urbain va demander une prise en compte de multiple décisions. Ce problème deviendra critique lorsque des véhicules automatisés pourront se déplacer au même titre que les conducteurs humains. Modifier son comportement engage une responsabilité qui pose des problèmes légaux mais également éthiques (Hevelke, Nida-Rümelin, 2014). Goodall (2014) justifie ce besoin d'éthique et répond aux critiques les plus fréquentes. Par exemple, certaines concernent le fonctionnement du véhicule et du trafic généré. Ainsi, le véhicule est considéré comme parfait, au regard d'un conducteur humain, et ne peut donc pas être responsable d'accident. A juste titre, l'auteur souligne la nécessité de prendre en compte une part d'aléas comme un objet sur la chaussée ou la traversée impromptue d'un piéton ou animal. Dans toutes ces situations, une décision du respect ou non du comportement prévu devra être envisagée avec la résolution éventuelle d'un conflit éthique. Le problème demeure que la décision soit prise localement par le véhicule ou dans un poste de régulation.

Ce questionnement sur les risques de respect de la privacité des informations et de la prise de décisions autonomes sont importants pour les chercheurs du domaine multi-agent. Nous montrons dans la section suivante comment des SMA sont utilisés afin de permettre localement à un conducteur d'apprécier la qualité des informations qu'il utilise (5.2.1) et comment un agent peut décider de ne pas respecter la règle commune (5.2.2).

\subsection{Illustration de l'utilisation de concepts agents face aux risques}

\subsubsection{Faciliter le partage de croyances}

Le projet «PLAteforme de sImulation d'Interaction entre entités MOBiles (Plaiimob) », dans le cadre du «Campus International sur la Sécurité et l'Intermodalité dans les Transports (CISIT) », (Markiewicz, 2010) vise à étudier le comportement des conducteurs relativement aux informations reçues dans des véhicules connectés (Adam et al., 2012). Dans ce modèle, le réseau routier est représenté par un graphe orienté et pondéré, le poids représente la vitesse maximale autorisée ou effective (déduite suite à l'observation de ralentissement) est fait l'objet des croyances.

La communication des croyances présuppose classiquement une prise en compte de la notion de confiance envers celles-ci. Du fait de la dynamique de l'environnement dans le cas précis du trafic routier, il n'est pas envisageable de supposer que toutes les entités communiqueront aux autres des croyances vérifiables. Le partage partiel des croyances et la non prise en compte d'informations sur les émetteurs limitent les risques de non respect de la confidentialité des informations. L'agent recevant différentes croyances à propos d'un même objet doit être capable de sélectionner la croyance la plus probable par rapport à l'état de ses connaissances. 
La communication de croyances entre des agents mobiles (i.e., les véhicules) évoluant dans un réseau peut être effectuée : directement (i.e., d'agent à agent) lorsqu'ils sont assez proches pour percevoir les messages; ou indirectement au travers de l'environnement (généralement en déposant des croyances sur les nœuds du réseau). Un troisième mode d'échange est également proposé dans lequel des agents non mobiles (e.g., des capteurs positionnés sur les feux de signalisation) sont placés sur les nœuds du réseau, communiquent avec les agents mobiles, et peuvent également inter-agir entre eux. Dans ce cadre, un nœud du réseau est non seulement doté de capacité de stockage de croyances. Mais, il peut aussi être considéré comme pro-actif en ayant la capacité de choisir les informations à communiquer ainsi que leurs destinataires (e.g., les nœuds voisins et les agents mobiles présents sur le nœud courant). Ce mode de communication via les nœuds communicants facilite la propagation d'informations à travers le réseau.

$\mathrm{Au}$ vu de l'état dynamique du trafic routier, les croyances sont rapidement obsolètes. Un coefficient de dégradation s'applique sur la confiance accordée à une croyance. Celui-ci est propre à chaque agent, représentant ainsi les différents comportements de conducteur humain. Ainsi un conducteur expert ou suspicieux placera un coefficient de dégradation élevé pour oublier plus rapidement la croyance reçue et revenir ainsi à ses croyances propres; au contraire, un conducteur novice ou confiant gardera plus longtemps en mémoire la croyance transmise par un pair. Cette réflexion sur le concept d'évolution de la confiance des croyances a été initialement proposée pour améliorer le temps d'acquisition dans les algorithmes d'apprentissage par renforcement avec oubli (Yen, Hickey, 2004).

Comme nous l'avons déjà évoqué, un agent peut posséder plusieurs croyances à propos d'un même objet, mais avec des valeurs différentes. Ce principe a été établi car il n'est pas possible pour un conducteur de faire pleinement confiance à la perception du dernier agent conducteur croisé : celui-ci peut avoir mal perçu un événement ou l'information qu'il a perçue n'était que ponctuelle, dû à un environnement fortement dynamique. Un agent effectue donc un choix parmi ses croyances, selon le principe qu'une croyance a d'autant plus de chance d'être sélectionnée que son niveau de confiance est élevé. Ainsi, comme le montre les différentes expérimentations (Adam et al., 2012), la majorité des agents évitent une route qu'ils croient perturbée, d'autre agents décident en revanche de tenter la route afin d'invalider, ou de confirmer, l'information signalant la perturbation.

La figure 4, extraite d'un simulateur réalisé avec la plateforme JADE, illustre une adaptation du comportement de véhicules autonomes. Un graphe représentant la ville de Toulouse a été extrait de la base Open Street Map (OSM) ${ }^{8}$. Dans cet exemple les véhicules doivent trouver le plus court chemin (en temps) entre deux endroits. Initialement, le chemin suivi est celui présent en (a). Un véhicule détectant un événement transmet l'information correspondante. Le message est perçu par les véhicules sui-

8. OSM (http://www.openstreetmap.org/) est un projet public permettant d'accéder aux cartes détaillées des villes du globes. 


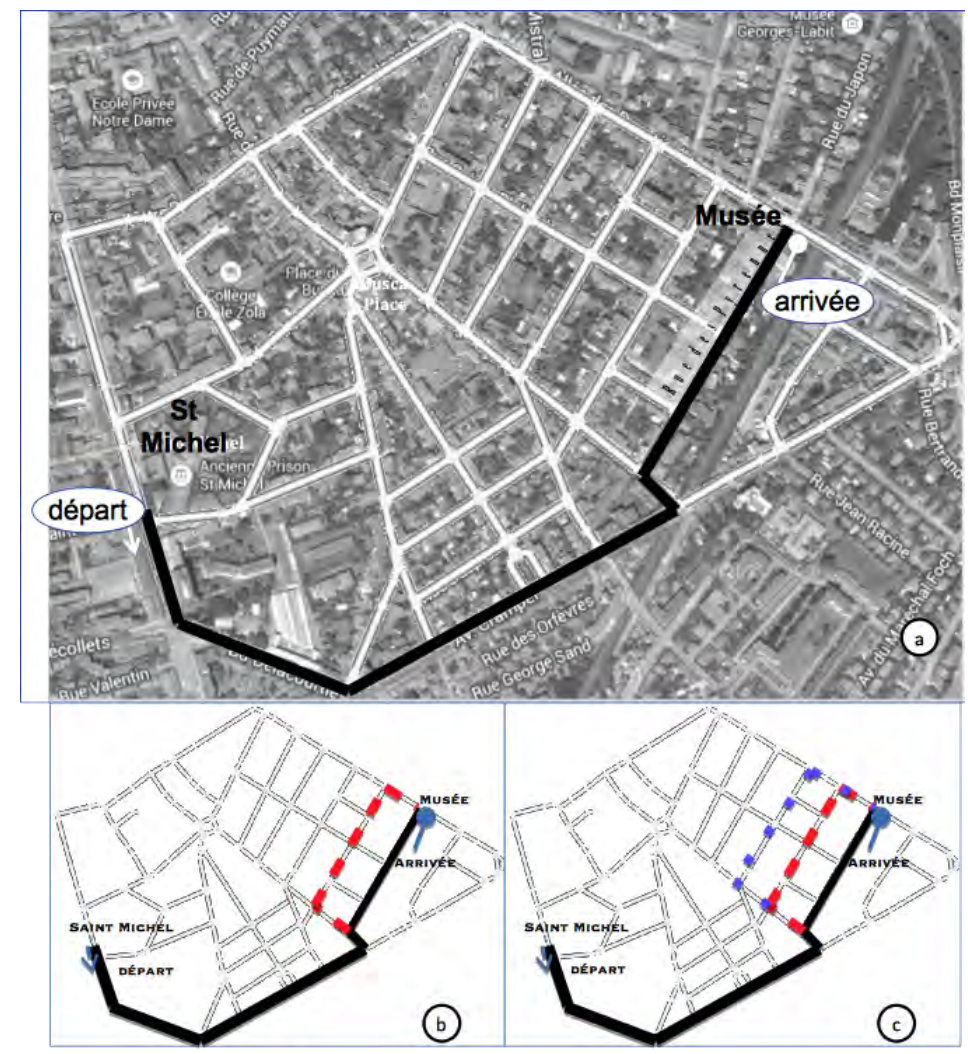

FIGURE 4. Recherche d'itinéraires dans la ville de Toulouse suite à une perturbation (plateforme Jade)

vants qui adaptent leurs trajets (b). De même un nouveau problème détecté sur la route amène les véhicules à choisir une troisième solution (c). Cependant, tous les véhicules ne cherchent pas à prendre le même chemin. La sélection des croyances sur l'état des routes est dépendante de leurs fiabilités et donc de leurs anciennetés. Quelques agents tentent épisodiquement d'utiliser la première ou la seconde route. Ces comportements permettent ainsi de détecter le retour à un état normal.

Cette communication de croyances permet ainsi un partage plus équitable des ressources (des routes) : lorsque trop d'agents utilisent la même route, la vitesse sur celleci est réduite (principalement lié au ralentissement au carrefour). Cette information est propagée et les agents se répartissent alors afin de fluidifier le trafic. Ce mécanisme de partage de croyances volatiles a également été testé sur des robots physiques autonomes (caractérisant des véhicules) qui se déplacent sur des voies spécifiques pour 
atteindre leurs propres destinations, réagissent correctement à des perturbations sur leurs axes de circulation (Ketenci et al., 2013).

\subsubsection{Faciliter l'usage des normes}

Généralement, une norme désigne un ensemble de règles et de principes appliqués à une entité et décrivant comment cette entité doit se comporter. En d'autres termes, les normes fournissent la manière de coordonner un ensemble d'agents selon différentes manières : par exemple, par des lois sociales de Shoham et Tennenholtz (1995), par l'influence de la norme sur la prise de décision individuelle dans une organisation (Gâteau et al., 2005), ou par la possibilité qu'ont les agents dont la rationalité repose sur le modèle «Belief-Desire-Intention $(\mathrm{BDI})$ » à pouvoir violer des normes (Dignum et al., 2000).

Dans un contexte de simulation de trafic routier, l'ensemble des règles composant le « code de la route» peut être vu comme une norme régissant le système de trafic. Un comportement non-normatif, c'est-à-dire un comportement ne respectant pas les normes du système, apparaît comme pertinent dans le cadre de cette application. En effet, nous pouvons facilement donner des exemples où les conducteurs humains ne respectent pas obligatoirement le code de la route (i.e., la norme). De nombreux travaux en psychologie de la conduite montrent en effet qu'un conducteur humain ne respecte pas toujours le code de la route et va même jusqu'à développer ses propres règles informelles de conduite. La formation de cette propre connaissance peut être le résultat de compromis entre ses différentes valeurs, et donc de son éthique. Par exemple, le respect des véhicules prioritaires ou la relation à la prise de risques mettent en jeu des valeurs personnelles des conducteurs. En revanche, les pénalités introduites également dans la communauté SMA (Gâteau et al., 2005) en cas de non respect des normes, ne semblent pas pertinentes pour ce type d'application.

Dans ce cadre, Doniec et al. (2006) définissent un ensemble de règles contextuelles permettant d'accorder une certaine tolérance à l'application stricte du code de la route. Comme nous l'avons déjà évoqué, ces règles de violation de la norme expriment l'idée que l'agent simulé puisse agir avec un comportement non normatif (proche d'un conducteur humain), en estimant qu'il n'y aura pas de risque de collision. Cependant, ce comportement ne nous garantit plus un flux global cohérent du trafic routier. Rappelons que le code de la route a été introduit non seulement pour empêcher des collisions entre véhicules; mais aussi pour éviter des situations d'interblocages. Comme nous l'avons par ailleurs évoqué, il apparait donc nécessaire de doter les agents de capacités pour anticiper de telles situations afin de pouvoir mâ̂triser la violation éventuelle de normes par les agents (Doniec et al., 2008). Notons aussi que cette capacité d'anticipation a aussi été proposée dans un autre contexte lié au trafic autoroutier (El Hadouaj et al., 2000). Dans la vie réelle, les conducteurs humains anticipent également les comportements des autres afin d'éviter des situations dangereuses : celles-ci dépendent essentiellement de leur expertise dans la conduite automobile. 


\section{Conclusion}

Des systèmes de transport intelligents (STI) sont l'un des « grands » enjeux sociétaux, à la fois en termes économiques, de développements durables ou plus simplement en termes de qualité de vie des citoyens. Comme nous l'avons déjà exprimé, les STI sont des systèmes complexes, et par nature distribués et ouverts; et comme nous l'avons illustré par les travaux présentés dans cet article, l'approche multi-agent est judicieuse pour étudier ou développer de tels systèmes. Ainsi, de notre point de vue, le paradigme multi-agent a atteint une maturité en termes de recherche lui permettant d'être validé dans des contextes opérationnels.

Dans cet article, notre objectif a consisté à proposer un panorama des principales problématiques soulevées par les STI, et comment les systèmes multi-agents pouvaient contribuer à la recherche de solutions. Dans la deuxième section, nous avons ainsi présenté le domaine des STI en mettant en évidence la diversité des sujets traités (e.g., la sécurité, la consommation d'énergie, la pollution) pour les transports de biens ou de voyageurs (e.g., billettique, logistique) jusqu'à la gestion des infrastructures existantes (e.g., régulation) et son interaction avec les usagers (e.g., véhicules connectés). Nous avons ainsi insisté sur les SMA comme une approche pouvant répondre à la conception de tels systèmes. Nous avons ensuite proposé dans la troisième section, l'usage des SMA comme une solution à la prise en compte d'un environnement complexe. Nous avons illustré ces difficultés par des exemples issus du domaine des Systèmes d'Information Voyageurs. Nous avons ainsi montré la difficulté d'exploiter une très grande masse d'informations dynamiques (e.g., cas de la gestion de parking) ou hétérogènes (e.g., cas de la prise en compte d'informations personnalisées), notamment des informations voyageurs dans un contexte multimodal. La section suivante s'est attachée au problème du passage à l'échelle des STI. Nous avons ainsi montré que les STI peuvent surmonter cet aspect très délicat, dans la manière de proposer des mécanismes de coordination. Enfin, la dernière section a envisagé les implications de la prise en compte des risques dans un STI, par exemple, dans l'utilisation d'agents autonomes en interaction avec des êtres humains. Nous avons également porté un intérêt pour les aspects éthiques (e.g., usage des normes) et le respect de la vie privée.

Comme nous l'avons montré, le domaine des STI est riche et complexe et d'autres problématiques sont à considérer. Ainsi l'importance des volumes de données à traiter et la sécurité sont d'autres exemples de problématiques pour lesquels les SMA pourraient apporter des contributions et l'étude de ces dernières constituent une de nos perspectives.

Pour conclure, nous pensons que la problématique STI soulève de nombreuses difficultés auxquelles les travaux de recherche dans les différents domaines peuvent apporter des éléments de réponse. Pour notre part, nous avons axé notre discours sur des approches multi-agents qui de par leur hypothèses peuvent contribuer à ce domaine. Il est également important de souligner que ce papier n'a pas vocation à présenter (de manière exhaustive) tous les travaux de recherche dans ce domaine; mais plutôt de proposer quelques travaux illustrant la richesse des problématiques à envisager. 


\section{Remerciements}

Les auteurs remercient les relecteurs anonymes pour leurs remarques constructives, qui ont permmis d'améliorer notre article.

\section{Glossaire}

BDI Belief-Desire-Intention

CISIT Campus International sur la Sécurité et l'Intermodalité dans les Transports eThicAa eThique et Agents autonomes

FIPA Foundation for Intelligent Physical Agents

IoV Internet of Vehicles

LUMD Logistique Urbaine Mutualisée et Durable

NHTSA National Highway Traffic Safety Administration

NTIC Nouvelles Technologies de l'Information et des Communications

OSM Open Street Map

OWL Ontology Web Language

Plaiimob PLAteforme de sImulation d'Interaction entre entités MOBiles

RDF Resource Description Framework

RFID Radio Frequency Identification

SAE Système d'Aide à l'Exploitation

SCATS Sydney Coordinated Adaptive Traffic System

SCOOT Spit Cycle Offset Optimisation Technique

SIV Système d'Information des Voyageurs

SMA Système Multi-Agent

STI Système de Transport Intelligent

VANETs Vehicular Ad Hoc Networks

\section{Bibliographie}

Adam E., Mandiau R., Grislin-Le Strugeon E. (2012, 28-30 March). Multilevel MAS architecture for vehicles knowledge propagating. In J. B. Pérez et al. (Eds.), Highlights on practical applications of agents and multi-agent systems - 10th international conference on practical applications of agents and multi-agent systems, PAAMS 2012 special sessions, vol. 156, p. 1-8. Salamanca, Spain, Springer.

Anderson J. M., Nidhi K., Stanley K. D., Sorensen P., Samaras C., Oluwatola O. A. (2014). Autonomous vehicle technology: A guide for policymakers. Rand Corporation.

Ando Y., Fukazawa Y., Masutani O., Iwasaki H., Honiden S. (2006, May 8-12). Performance of pheromone model for predicting traffic congestion. In H. Nakashima, M. P. Wellman, G. Weiss, P. Stone (Eds.), 5th international joint conference on autonomous agents and multiagent systems (AAMAS), p. 73-80. Hakodate, Japan, ACM. 
24 RIA. Volume $\mathrm{xx}-\mathrm{n}^{0} \mathrm{xx}-\mathrm{xx} / 201 \mathrm{x}$

Balbo F., Pinson S. (2010). Using intelligent agents for transportation regulation support system design. Transportation Research part C: emerging technologies, vol. 18, $\mathrm{n}^{\circ}$ 1, p. 140-156.

Banâtre M., Couderc P., Pauty J., Becus M. (2004, Sept. 13-16). Ubibus: Ubiquitous computing to help blind people in public transport. In S. A. Brewster, M. D. Dunlop (Eds.), Mobile human-computer interaction - mobile HCI 2004, 6th international symposium, vol. 3160 , p. 310-314. Glasgow, UK, Springer.

Bazzan A. L. C. (2005). A distributed approach for coordination of traffic signal agents. Journal of Autonomous Agents and Multi-Agent Systems, vol. 10, p. 131-164.

Bazzan A. L. C., Klügl F. (2014). A review on agent-based technology for traffic and transportation. Knowledge Eng. Review, vol. 29, n 3, p. 375-403.

Bessghaier N., Zargayouna M., Balbo F. (2012, December 4-7). Online localized resource allocation application to urban parking management. In IEEE/WIC/ACM international conferences on Intelligent Agent Technology (IAT), p. 67-74. Macau, China, IEEE.

Bhouri N., Balbo F., Pinson S. (2012). An agent-based computational approach for urban traffic regulation. Progress in Artificial Intelligence, vol. 1, no 2, p. 139-147.

Bonhomme A., Mathieu P., Picault S. (2015, June 3-4). Trafficgen: A flexible tool for informing agent-based traffic simulations with open data. In Y. Demazeau, K. S. Decker, J. B. Pérez, F. de la Prieta (Eds.), Advances in practical applications of agents, multi-agent systems, and sustainability: The PAAMS collection - 13th international conference (PAAMS), vol. 9086, p. 259-262. Salamanca, Spain, Springer.

Boy G. (2014). From automation to tangible interactive objects. Annual Reviews in Control, vol. $38, \mathrm{n}^{\circ} 1$, p. $1-11$.

Camus B., Bourjot C., Chevrier V. (2015). Considering a multi-level model as a society of interacting models: Application to a collective motion example. Journal Artificial Societies and Social Simulation, vol. 18, $\mathrm{n}^{\circ} 3$.

Casey C. (2003). Real-time information: Now arriving. Metro, vol. 99, $\mathrm{n}^{\circ} 3$.

Champion A. (2003). Mécanisme de coordination multi-agent à base de jeux: application à la simulation comportementale de trafic routier en situation de carrefour. Thèse de doctorat non publiée, Université de Valenciennes, France.

Chen B., Cheng H. H., Palen J. (2009). Integrating mobile agent technology with multi-agent systems for distributed traffic detection and management systems. Transportation Research Part C: Emerging Technologies, vol. 17, n 1, p. 1-10.

Coyle L., Cunningham P., Hayes C. (2002, Sept. 4-7). A case-based personal travel assistant for elaborating user requirements and assessing offers. In S. Craw, A. D. Preece (Eds.), Advances in Case-Based Reasoning, 6th European Conference, ECCBR, vol. 2416, p. 505518. Aberdeen, Scotland, UK, Springer.

Dafflon B., Gechter F. (2014). Making decision with reactive multi-agent systems: A possible alternative to regular decision processes for platoon control issue. Research in Computing Science, vol. 86, p. 101-112.

Delot T., Cenerario N., Ilarri S. (2010). Vehicular event sharing with a mobile peer-to-peer architecture. Transportation Research part C: Emerging Technologies, vol. 18, no 4, p. 584 598. 
Delot T., Cenerario N., Ilarri S., Lecomte S. (2009, Sept. 2-4). A cooperative reservation protocol for parking spaces in vehicular ad hoc networks. In 6th International conference on Mobile Technology, Applications, and Systems, Mobility conference. Nice, France, ACM.

DGITM. (2011, Juin). Les systèmes de transport intelligent en france (SIT). Rapport technique. Paris, France, Direction Générale des Infrastructures, des Transports et de la Mer, Ministère de L'Ecologie, du Développement durable, des Transports et du Logement.

Dignum F., Morley D. N., Sonenberg L., Cavedon L. (2000, July 10-12). Towards socially sophisticated BDI agents. In 4th International Conference on Multi-Agent Systems, ICMAS, p. 111-118. Boston, MA, USA, IEEE Computer Society.

Dokic J., Muller B., Meyer G. (2015, April 1st). European Roadmap Smart Systems for Automated Driving. Rapport technique. Berlin.

Doniec A., Mandiau R., Espié S., Piechowiak S. (2006, Dec. 18-22). Non-normative behaviour in multi-agent system: some experiments in traffic simulation. In IEEE/WIC/ACM International Conference on Intelligent Agent Technology (IAT), p. 30-36. Hong Kong, China, IEEE Computer Society.

Doniec A., Mandiau R., Piechowiak S., Espié S. (2008). Anticipation based on constraint processing in a multi-agent context. Journal of Autonomous Agents and Multi-Agent Systems, ISSN 1387-2532, Springer Netherlands, vol. 17, $\mathrm{n}^{\circ}$ 2, p. 339-361.

El Hadouaj S., Drogoul A., Espié S. (2000, July). How to combine reactivity and anticipation: the case of the conflicts resolution in a simulated road traffic? In S. Moss, P. Davidsson (Eds.), Multi-Agent-Based Simulation: Second international workshop (MABS), vol. 1979, p. 82-96. Boston, MA, USA, Springer.

Ernst T., Nebehaj V., Soråsen R. (2009, Oct. 20-22). CVIS: CALM proof of concept preliminary results. In 9th International Conference on Intelligent Transport Systems Telecommunications (ITST), p. 80-85.

Espié S. (1995). Archisim, multi-actor parallel architecture for traffic simulation. In Proceedings of the second world congress on intelligent transport systems. Yokohama, Japan.

EU. (2012). Innover pour un système de transport compétitif et économe en ressources. Rapport technique. Union Européenne.

EU. (2014a, Nov.). Climate action: Building a world we like, with a climate we like. Rapport technique. European Union.

EU. (2014b). Hozizon 2020 : Le programme-cadre de l'UE pour la recherche et l'innovation. Rapport technique. Union Européenne.

Faure L., Burlat P., Montreuil B., Marquès G. (2014). A modelling approach of pooled urban consolidation centers. In 2014 gis urban modelling symposium.

Gâteau B., Boissier O., Khadraoui D., Dubois E. (2005, Dec. 7-8). Moise ${ }^{\text {Inst }}$ : An organizational model for specifying rights and duties of autonomous agents. In M. P. Gleizes, G. A. Kaminka, A. Nowé, S. Ossowski, K. Tuyls, K. Verbeeck (Eds.), Third EUropean workshop on Multi-Agent Systems (EUMAS), p. 484-485. Brussels, Belgium, Koninklijke Vlaamse Academie van Belie voor Wetenschappen en Kunsten.

Gechter F., Contet J., Galland S., Lamotte O., Koukam A. (2012). Virtual intelligent vehicle urban simulator: Application to vehicle platoon evaluation. Simulation Modelling Practice and Theory, vol. 24, p. 103-114. 
RIA. Volume $\mathrm{xx}-\mathrm{n}^{0} \mathrm{xx}-\mathrm{xx} / 201 \mathrm{x}$

Giannopoulos G. A. (2004). The application of information and communication technologies in transport. European Journal of Operational Research, vol. 152, no 2, p. 302-320.

Glancy D. J. (2012). Privacy in autonomous vehicles. Santa Clara Law Review, vol. 52, p. 1171-1239.

Goodall N. (2014). Machine ethics and automated vehicles. In G. Meyer, S. Beiker (Eds.), Road vehicle automation, p. 93-102. Springer.

Goodall N., Smith B., Park B. (2013). Traffic signal control with connected vehicles. Transportation Research Record: Journal of the Transportation Research Board, $\mathrm{n}^{\circ}$ 2381, p. 65-72.

Guériau M., Billot R., Faouzi N. E., Hassas S., Armetta F. (2015, Jan. 25-30). Multi-agent dynamic coupling for cooperative vehicles modeling. In B. Bonet, S. Koenig (Eds.), Twentyninth AAAI conference on Artificial Intelligence, p. 4276-4277. Austin, Texas, USA, AAAI Press.

Guerrien B. (1997). La théorie des jeux. Economica. Paris.

Guessoum Z., Mandiau R., Mathieu P., Boissier O., Glize P., Hamri A. et al. (2012). Sytèmes multi-agents et simulation. In J.-M. Ogier, F. Sedes, P. Marquis (Eds.), Information, Interaction, Intelligence, le point sur le I3, p. 76-120. Toulouse, France, Cepadues Editions.

Hall F. (1997). Traffic stream characteristics. In N. Gartner, C. Messer, A. Rathi (Eds.), Traffic flow theory: A State of the Art Report, chap. 2. Oak Ridge National Laboratory.

Hette G., Estivie S., Adam E., Mandiau R. (2012). Ré-allocation dynamique de tâches pour un réseau de transport. Revue d'Intelligence Artificielle, vol. 26, nº 6, p. 709-734.

Hevelke A., Nida-Rümelin J. (2014). Responsibility for crashes of autonomous vehicles: an ethical analysis. Science and engineering ethics, vol. 21, n 3, p. 619-630.

Hunt P., Robertson D., Bretherton R., Royle M. C. (1982). The scoot on-line traffic signal optimisation technique. Traffic Engineering \& Control, vol. 23, nº 4.

Hussain I., Knapen J., Khan M. A., Bellemans T., Janssens D., Wets G. (2015). Agent-based negotiation model for long-term carpooling: A flexible mechanism for trip departure times. Urban Transport XXI, vol. 146, p. 461.

Jennings N. (1996). Coordination techniques for distributed artificial intelligence. In Foundations of distributed artificial intelligence, p. 187-210. Wiley.

Jorion P. (1989). Principes des systèmes intelligents. Masson.

Kakkasageri M., Manvi S., Pitt J. (2013). Cognitive agent based critical information gathering and dissemination in vehicular ad hoc networks. Wireless personal communications, vol. 69, $\mathrm{n}^{\circ} 4$, p. $1107-1129$.

Ketenci U., Adam E., Grislin E., Mandiau R. (2013, Dec. 12-13). Volatile knowledge for mobile agents : application to autonomous vehicles management. In 11th EUropean workshop on Multi-Agent Systems (EUMAS). Toulouse, France. (Short paper)

Koukoumidis E., Martonosi M., Peh L.-S. (2012). Leveraging smartphone cameras for collaborative road advisories. IEEE Transactions on Mobile Computing, vol. 11, $\mathrm{n}^{\mathrm{o}}$ 5, p. 707-723.

Ksontini F., Mandiau R., Guessoum Z., Espié S. (2015). Affordance-based agent model for road traffic simulation. Autonomous Agents and Multi-Agent Systems, vol. 29, no 5 , p. 821-849. 
Lesser V. (1998). Reflections on the nature of multi-agent coordination and its implications for an agent architecture. Autonomous Agents and Multi-Agent Systems, vol. 1, p. 89-111.

Li Q., Chen L., Li M., Shaw S.-L., Nuchter A. (2014). A sensor-fusion drivable-region and lanedetection system for autonomous vehicle navigation in challenging road scenarios. IEEE Transactions on Vehicular Technology, vol. 63, n 2, p. 540-555.

Lieberman E., Rathi A. (1997). Traffic simulation. In N. Gartner, C. Messer, A. E. Rathi (Eds.), Traffic flow theory: A State of the Art Report, chap. 10. Oak Ridge National Laboratory.

Lowrie P. (1990). Scats, sydney co-ordinated adaptive traffic system: A traffic responsive method of controlling urban traffic.

Luo J., Hubaux J. (2005). A survey of research in inter-vehicle communications. In K. Lemke, C. Paar, M. Wolf (Eds.), Embedded Security in Cars - Securing Current and Future Automotive IT Applications, p. 111-122. Springer Verlag.

Luo Y. (2007). A coopetition perspective of global competition. Journal of World Business, vol. $42, \mathrm{n}^{\circ} 2$, p. 129-144.

Malone T. (1998). What is coordination theory? In National science foundation coordination theory workshop.

Mandiau R., Champion A., Auberlet J., Espié S., Kolski C. (2008). Behaviour based on decision matrices for a coordination between agents in urban traffic simulation. Applied Intelligence, vol. $28, \mathrm{n}^{\circ} 2$, p. 121-138.

Mandiau R., Piechowiak S., Doniec A., Espié S. (2012). Agent-oriented road traffic simulation. In S. Hammadi, M. Ksouri (Eds.), Advanced mobility and transport engineering, p. 1-30. ISTE-Wiley.

Markiewicz E. (2010). International campus on safety and intermodality in transportation activity report,. Rapport technique. Valenciennes, France.

Moor J. M. (2006). The nature, importance, and difficulty of machine ethics. IEEE Intelligent Systems, vol. 21, no 4, p. 18-21.

Moraitis P., Petraki E., Spanoudakis N. I. (2005, Dec. 7-8). An agent-based system for infomobility services. In M. P. Gleizes, G. A. Kaminka, A. Nowé, S. Ossowski, K. Tuyls, K. Verbeeck (Eds.), Third EUropean workshop on Multi-Agent Systems (EUMAS, p. 224 235. Brussels, Belgium, Koninklijke Vlaamse Academie van Belie voor Wetenschappen en Kunsten.

Nangeroni C. (2010). Stationnement intelligent : à la bonne place au bon moment. Ville, rail et transport, $\mathrm{n}^{\circ} 507$, p. 30-33.

Nasim R., Kassler A. (2012, Dec. 3-4). Distributed architectures for intelligent transport systems: A survey. In Second symposium on Network Cloud Computing and Applications (NCCA), p. 130-136. London, GB.

Núñez-Suárez J., O’Sullivan D., Brouchoud H., Cros P., Moore C., Byrne C. (2000). Experiences in the use of FIPA agent technologies for the development of a personal travel application. In 4th international conference on Autonomous Agent, p. 357-364.

Oliveira K. M. de, Bacha F., Houda M., Abed M. (2013). Transportation ontology definition and application for the content personalization of user interfaces. Expert Systems with Applications., vol. 40, no 8, p. 3145-3159. 
Pandit K., Ghosal D., Zhang H. M., Chuah C.-N. (2013). Adaptive traffic signal control with vehicular ad hoc networks. Vehicular Technology, IEEE Transactions on, vol. 62, no 4 , p. $1459-1471$.

Papageorgiou M., Ben-Akiva M., Bottom J., Bovy P. H., Hoogendoorn S., Hounsell N. B. et al. (2007). ITS and traffic management. In C. Barnhart, G. Laporte (Eds.), Handbooks in operations research and management science, vol. 14, p. 715-774. Elsevier.

Paruchuri P., Pullalarevu A. R., Karlapalem K. (2002, July 15-19). Multi agent simulation of unorganized traffic. In 1st internationnal conference on Autonomous Agents and Multiagent Systems (AAMAS), p. 176-183. Bologne, Italy, ACM.

Petit-Rozé C., Anli A., Grislin-Le Strugeon E., Abed M., Uster G., Kolski C. (2004). Système d'information transport personnalisée à base d'agents logiciels. Génie logiciel, vol. 70, p. 29-38.

Poslad S. (2007). Specifying protocols for multi-agent systems interaction. ACM Transactions on Autonomous and Adaptive Systems TAAS, vol. 2, no 4. Consulté sur http://doi.acm.org/ $10.1145 / 1293731.1293735$

Prentice J. (1974). The evasive action decision in an intersection accident: a game theory approach. Journal of Safety Research, vol. 6, no 4, p. 146-149.

Radu A., Rothkrantz L., Novak M. (2013). Digital traveler assistant. In J.-L. Ferrier, A. Bernard, A. Gusikhin, K. Madani (Eds.), Informatics in Control, Automation and Robotics, vol. 174, p. 101-114. Springer.

Rafiq G., Talha B., Patzold M., Gato Luis J., Ripa G., Carreras I. et al. (2013). What' s new in intelligent transportation systems?: An overview of european projects and initiatives. IEEE Vehicular Technology Magazine, vol. 8, nº 4, p. 45-69.

Reece D. A., Shafer S. A. (1993). A computational model of driving for autonomous vehicles. Transportation Research, vol. 27, $\mathrm{n}^{\mathrm{o}}$ 1, p. 23-50.

Repenning A., Ioannidou A. (2006). Mobility agents: Guiding and tracking public transportation users. In Working conference on Advanced Visual Interfaces, p. 127-134. New York, NY, USA, ACM.

Ruskin H. J., Wang R. (2002, April 21-24). Modelling traffic flow at an urban unsignalized intersection. In P. M. Sloot, A. G. Hoekstra, K. C. Tan, J. J. Dongarra (Eds.), International Conference on Computational Science (ICCS), vol. 2329, p. 381-390. Amsterdam, Netherlands, Springer.

Russell S. J., Dewey D., Tegmark M. (2015). Research priorities for robust and beneficial artificial intelligence. AI Magazine, vol. 36, no 4. Consulté sur http://www.aaai.org/ojs/ index.php/aimagazine/article/view/105-114

Saunier J., Balbo F., Pinson S. (2014). A formal model of communication and context awareness in multiagent systems. Journal of Logic, Language and Information, vol. 23, n 2, p. 219 247.

Shoham Y., Tennenholtz M. (1995). On social laws for artificial agent societies: Off-line design. Artificial Intelligence, vol. 73, no 1-2, p. 231-252.

Shoup D. C. (2006). Cruising for parking. Transport Policy, vol. 13, nº 6, p. 479-486. (Parking) 
Silberg G., Wallace R., Matuszak G., Plessers J., Brower C., Subramanian D. (2012). Selfdriving cars: The next revolution. White paper, KPMG LLP \& Center of Automotive Research.

Steinfeld A. (2010). Ethics and policy implications for inclusive intelligent transportation systems. In Second international symposium on quality of life technology.

Tlig M., Buffet O., Simonin O. (2014, 18-22 Aug.). Stop-free strategies for traffic networks: Decentralized on-line optimization. In T. Schaub, G. Friedrich, B. O'Sullivan (Eds.), ECAI 2014 - 21st european conference on artificial intelligence, including prestigious applications of intelligent systems (PAIS 2014), vol. 263, p. 1191-1196. Prague, Czech Republic, IOS Press.

Todts W., Calvo Ambel C. (2015, June). Road to 2030: how eu vehicle efficiency standards help member states meet climate targets. Rapport technique. European Union.

Trannois H., Lebrun A., Deleage J. (1998, March 23-25). A multi-agent framework for car traffic simulation. In Third international conference and exhibition on the Practical Application of Intelligent Agents and Multi-agents (PAAM). London, UK.

Vanderhaegen F., Chalmé S., Anceaux F., Millot P. (2006). Principles of cooperation and competition: application to car driver behavior analysis. Cognition, Technology \& Work, vol. 8, n $^{\circ} 3$, p. 183-192.

Vinyals M., Rodriguez-Aguilar J. A., Cerquides J. (2011). A survey on sensor networks from a multiagent perspective. The Computer Journal, vol. 54, nº 3, p. 455-470.

Vissim. (2005). Vissim 4.10. user manual. Rapport technique. PTV Planung Transport Verkehr AG: Karlsruhe, Germany.

Wen W. (2010). An intelligent traffic management expert system with RFID technology. International Journal of Expert Systems with Applications, vol. 37, nº 4, p. 3024-3035.

Wunderlich R., Liu C., Elhanany I., Urbanik T. (2008, Sept.). A novel signal-scheduling algorithm with quality-of-service provisioning for an isolated intersection. IEEE Transactions on Intelligent Transportation Systems, vol. 9, $\mathrm{n}^{\circ}$ 3, p. 536-547.

Xiong Y., Gan J., An B., Miao C., Bazzan A. L. C. (2015, July 25-31). Optimal electric vehicle charging station placement. In Q. Yang, M. Wooldridge (Eds.), Twenty-fourth International Joint Conference on Artificial Intelligence (IJCAI), p. 2662-2668. Buenos Aires, Argentina, AAAI Press.

Yen G. G., Hickey T. W. (2004). Reinforcement learning algorithms for robotic navigation in dynamic environments. ISA Transactions, vol. 43, no 2, p. 217-230.

Zargayouna M., Balbo F., Trassy J. S. (2006, Oct. 26-28). Agent information server: a middleware for traveler information. In O. Dikinelli, M. P. Gleizes, A. Ricci (Eds.), Engineering societies in the agents world vi: 6th international workshop (ESAW), vol. 3963, p. 14-28. Kusadasi, Turkey, Springer. 